\title{
Composite Hodgkin and Non-Hodgkin Lymphomas: Report of Two Cases with Unusual Presentations
}

Rami Nassabein ${ }^{1}$, Siham Fleifel ${ }^{2}$, Noha bejjani ${ }^{2}$, Hady Ghanem ${ }^{1}$, Dany Abi Gerges ${ }^{1}$, Roland De Wind ${ }^{3 *}$, Wassim Serhal ${ }^{4}$ and Hussein Farhat ${ }^{4}$

${ }^{1}$ Faculty of Medical Sciences, Department of Hematology-Oncology, Lebanese University, Lebanon

${ }^{2}$ Department of Pathology, Lebanese American University Medical Center (LAUMC), Lebanon

${ }^{3}$ Department of Pathology, Institute of Julet Bordet, Belgium

${ }^{4}$ Department of Laboratory Medicine, Lebanese American University Medical Center, Lebanon

\begin{abstract}
Composite lymphoma is a rare entity of lymphoma where two types of lymphoma are present synchronously. We recently encountered two cases of composite lymphomas that are not rare in the morphology and pathology only, but also rare in the site of involvement and presentation. In this report we present a case of composite lymphoma that presented as primary splenic lymphoma, the second case presented with $\mathrm{Gl}$ symptoms due to its presence in the colon.
\end{abstract}

Keywords: Hypercalcemia; Primary splenic lymphoma; Composite lymphoma

\section{Introduction}

Composite lymphoma is defined as coexistence of two types of lymphoma within the same organ at the same time of diagnosis. In composite lymphomas, the combination may be Hodgkin lymphoma with a Non-Hodgkin lymphoma (NHL) either B or T cells, or two different entities of NHLs. Primary Splenic Lymphoma, is a rare neoplasm of the spleen, comprising less than $2 \%$ of all the lymphomas [1]. It is defined as splenic disease (Diffuse large B cell lymphoma or low grade lymphoma) with possible hilar lymph node involvement without evidence of systemic lymphadenopathy [2]. In this report we represent two cases of composite lymphoma with Hodgkin lymphoma and NHL, B-cell high grade lymphomas with unusual presentation.

\section{Case 1}

An 83 year old male, with several co-morbidities including HTN,DM,CKD, COPD with 60 pack year cigarette smoking presented with more than 1 month history of constipation, fatigue, poor activity tolerance and about $6 \mathrm{~kg}$ weight loss over the past 6 months. Physical examination was significant for bilateral expiratory wheezes on lung auscultation, with no lymphadenopathy or hepatosplenomegaly. Neurological exam did not reveal any focal deficit. Initial workup revealed normocytic anemia Hb: $11 \mathrm{~g} / \mathrm{dL}$ MCV: 85 ESR: 99 at 1st hour. calcium: $13.7 \mathrm{mg} / \mathrm{dL}$ Albumin: $4 \mathrm{~g} / \mathrm{dL}$. Management of hypercalcemia was started with aggressive intravenous hydration, with furosemide added for the risk of fluid overload. At the same time, workup of hypercalcemia was done. PTH:18 (nl: $15-65 \mathrm{pg} / \mathrm{mL}$ ) and $25(\mathrm{OH})$ vitamin D: $24 \mathrm{ng} / \mathrm{mL}$. Serum and urine protein electrophoresis did not show a monoclonal protein. Hypercalcemia secondary to malignancy was suspected. CT scan of the chest, abdomen and pelvis showed multiple mediastinal and hilar subcentimeteric lymph nodes of which none reaching criteria of lymphadenopathy. No splenomegaly or hepatomegaly and no suspicious lesions on the bones. Since malignancy was suspected as the cause of hypercalcemia, FDG PET/ CT scan was done, which showed an intense and heterogeneous uptake in the spleen which measures $11.7 \mathrm{~cm}$, with FDG avid primary process occupies most of the spleen with SUV:14.5 with a splenic hilar LN 2.4 $\mathrm{cm}$ and SUV: 8.5 only (Figure 1).

Trying to avoid further invasive procedure, Bone marrow aspirate, Biopsy and flow cytommetry done did not reveal any abnormal cells invading the bone marrow. Thus, after decreasing calcium levels with the addition of pamidronate $30 \mathrm{mg}$, surgical risk assessment and immunization, patient underwent open splenectomy (Figure 2). On microscopic examination, the architecture of spleen is disrupted by the presence of wide cellular area surrounded by fibrosis with mixture of some eosinophils, histiocytes and a majority of large cells that are either immunoblast-like with irregular nuclear contour or with Hodgkin like features. The latter cells are binucleated or multinucleated with large eosinophilic nucleoli. On immunohistochemistry, the populations of large cells express partially CD 30 with heterogeneous intensity with the majority of cells markedly expressing CD 20. However, the cells that do not express CD 20 but are CD 30 positive, are weakly marked by anti-PAX- 5 and negative for CD 45 , corresponds to Hodgkin cells (Reed-Sternberg cells). CD 15 and EBV by EBRB probe are negative. CD 10 is expressed by minority of these large cells $(<30 \%)$ as compared
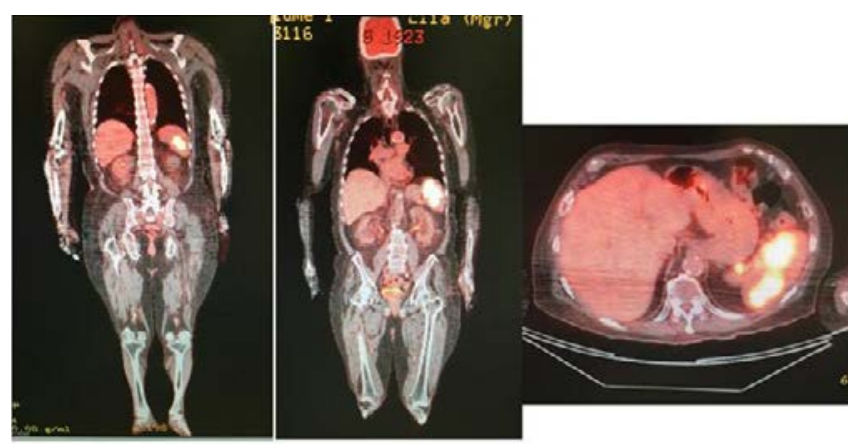

Figure 1: PET/CT of the patient showing isolated FDG avid spleen and splenic hilar Lymph node.

*Corresponding author: Wind RD, Department of Pathology, Institute of Julet Bordet, Belgium, Tel: 009613064454; E-mail: roland.dewind@bordet.be

Received August 08, 2017; Accepted October 31, 2017; Published November 15,2017

Citation: Nassabein R, Fleifel S, Bejjani N, Ghanem H, Gerges DA, et al. (2017) Composite Hodgkin and Non-Hodgkin Lymphomas: Report of Two Cases with Unusual Presentations. J Blood Lymph 7: 180. doi: 10.4172/2165-7831.1000180

Copyright: (c) 2017 Nassabein R, et al. This is an open-access article distributed under the terms of the Creative Commons Attribution License, which permits unrestricted use, distribution, and reproduction in any medium, provided the original author and source are credited. 
Citation: Nassabein R, Fleifel S, Bejjani N, Ghanem H, Gerges DA, et al. (2017) Composite Hodgkin and Non-Hodgkin Lymphomas: Report of Two Cases with Unusual Presentations. J Blood Lymph 7: 180. doi: 10.4172/2165-7831.1000180

Page 2 of 3

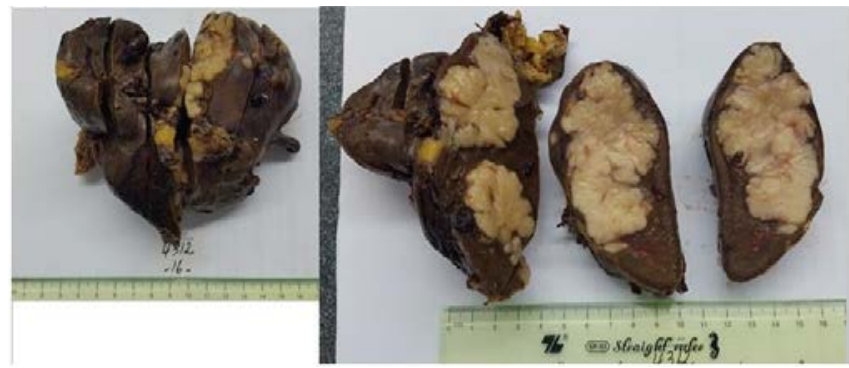

Figure 2: Grossly, the spleen measured $13 \times 9.5 \times 5 \mathrm{~cm}$ studded with large whitish nodules ranging from $0.5 \mathrm{~cm}$ to $7 \mathrm{~cm}$ occupying extensively the liver parenchyma.

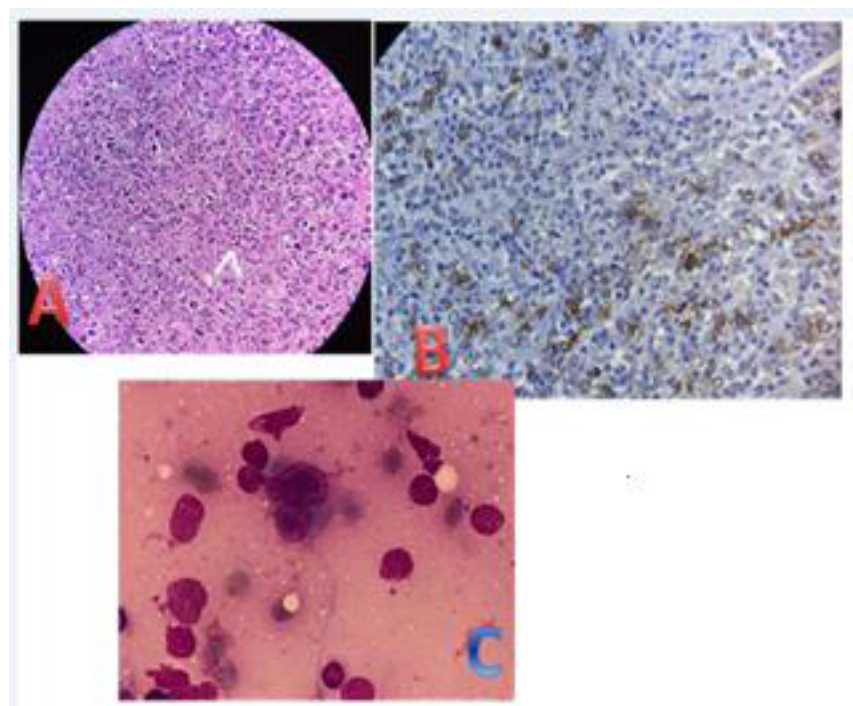

Figure 3: A- H\&E staining showing large cells, these cells are CD 30 positive as $B$. C is the cytology showing features of these cells that look like reed-sternberg cells.

to more expression of BCL-6 (>30\%). CD 23 highlights the rare residual lymphoid follicles (follicular dendritic cells) containing sometimes centers and identified by PD-1 and CXCL-13. CD 5 is expressed by small interstitial lymphocytes. Majority of large cells are marked by MUM-1. Ki 67 of CD 20 population is $50 \%$. These findings are in favor of composite lymphoma: Diffuse large B cell and classical Hodgkin Lymphoma rather than B-cell lymphoma, unclassifiable, with features intermediate between DLBCL and classical Hodgkin lymphoma (Figure 3). Patient had several post-op complications including ileus, pneumonia, and urinary retention secondary to orchitis for which he was hospitalized and received several courses of intravenous antibiotics.

\section{Case 2}

Our second case is a 47 year old female, known to have verruca valgaris on bilateral hands of long time duration, and history of about 4 months of anorexia, nausea, night sweats and weight loss of $10 \mathrm{~kg}$; presented with vomiting and abdominal pain. Physical examination was not significant except for left lower quadrant tenderness. A CT scan of the abdomen and pelvis was obtained. It showed circumferential wall thickening of the cecum $(17 \mathrm{~mm})$, extending over a length of almost $3 \mathrm{~cm}$, with narrowing of the lumen that remained patent with multiple enlarged mesenteric lymph nodes, mainly at the right colic and peri-cecal lymph nodes. A colonoscopy followed and revealed a large fungating mass in the cecum. PET-CT scan showed a mediastinal LN measuring $1.4 \mathrm{~cm}$ and SUV max of 5.7; with intense uptake at the cecum of $4.8 \mathrm{~cm}$ and SUV of 16.7 extending to the terminal ileum. Cluster of aortic LNs measuring $3 \times 1.8 \mathrm{~cm}$ with SUV 11.6. Bone marrow showed heterogeneous uptake. These findings are consistent with stage IV disease. Bone marrow biopsy was negative for malignant cells. Biopsy of the cecal mass, showed large neoplastic cells expressing CD30 and PAX 5. These cells are negative for CD 20, CD45, CD35, CD15 and cyclin D1. Another population of medium sized cells was positive for CD20. By flow cytommetry, CD 20 positive cells showed monoclonal Kappa expression that could not be revealed by immunohistochemistry (Figure 4 ). The large cells are positive for EBV by EBER. HHV-8 and CD 138 are negative in these cells which go against plasmablastic cells. These finding are suggestive of composite lymphoma of classical Hodgkin type and Non-Hodgkin lymphoma (marginal zone??) (Figures 5 and 6).

\section{Discussion}

Composite lymphomas are been described in literature and account about $1-4 \%$ of all lymphomas. Also primary splenic lymphomas exist,

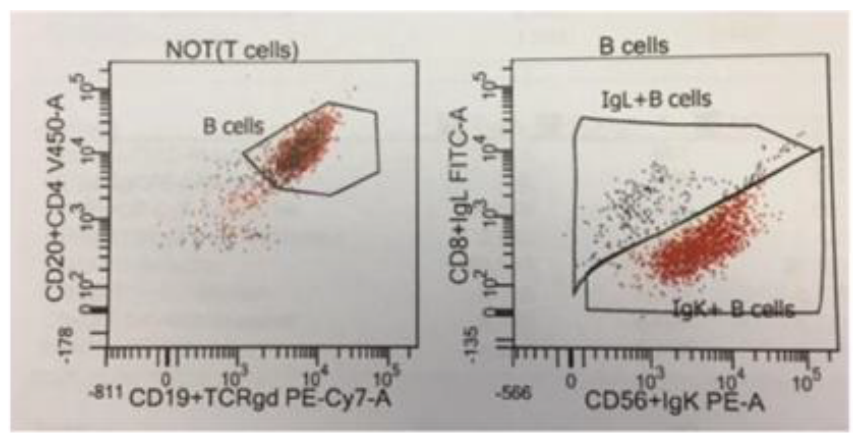

Figure 4: Flow cytommetry of CD 20 cells showing monoclonal kappa expression.

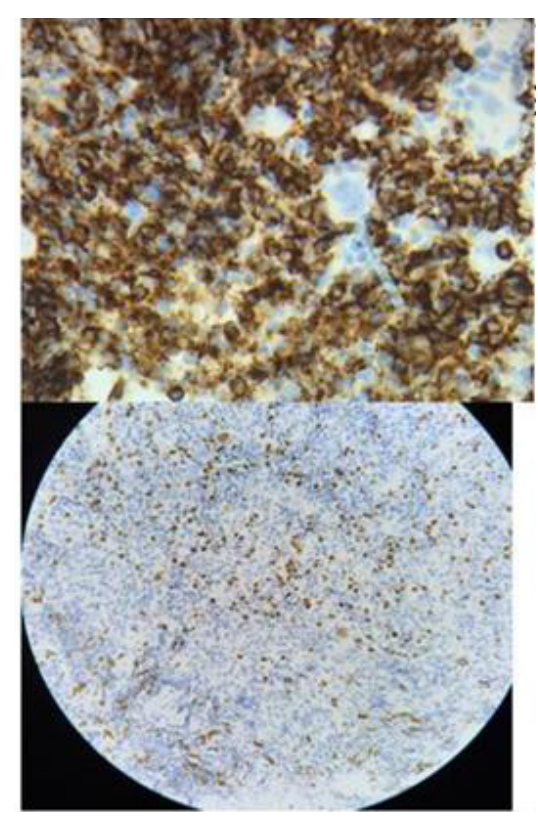

Figure 5: Large cells that are negative for $C D 45$ in the upper image and positive for CD 30 and PAX-5 in the lower image. 


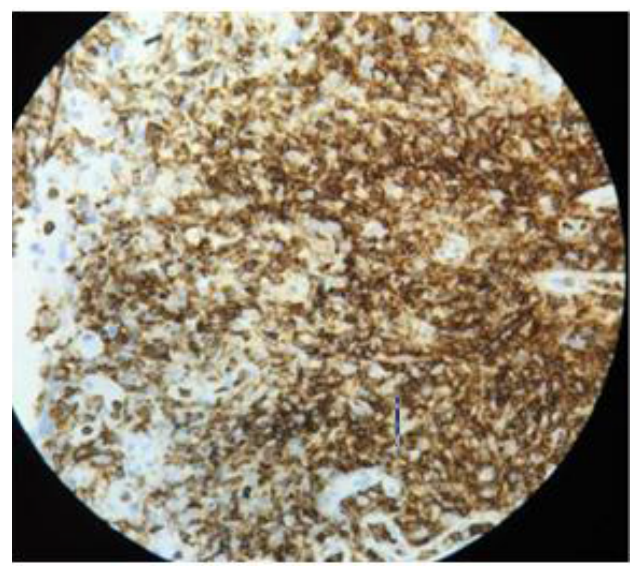

Figure 6: CD 20 positivity of the majority of cells of the biopsy of the colon.

but are very rare tumors that present with splenomegaly and cytopenia. Our first case is unique, as it was presented with hypercalcemia as paraneoplastic without evidence of cytopenia or splenomegaly. Even the CT scan done, unfortunately without contrast for chronic kidney disease did not reveal any changes or abnormal findings within the spleen parenchyma. The only finding was the FDG avid spleen with no evidence of lymphoma infiltration of the bone marrow. The diagnosis was reached as the majority of cases reported by splenectomy. In reviewing the literature, primary splenic lymphoma patients with diffuse large B cell lymphoma was described, but with different presentations including huge splenomegaly, cytopenias or part of systemic lymphoma [3]. Other reports described primary Hodgkin lymphomas of the spleen [4]. Composite lymphoma as primary splenic lymphoma or splenic involvement are rare cases and described in about 5 previous reported cases, but this is the second case in literature to our knowledge that present with primary splenic lymphoma and found to have Hodgkin and Non-Hodgkin type(large B-cell) [5]. In a review, majority of composite lymphomas with classical Hodgkin lymphoma and diffuse large B cell lymphomas are clonally related (originating from the same germinal B cell). If the two lymphomas were consecutive, then the two lymphomas are clonally unrelated. It was suggested that both lymphoma cells has the same mutation, but one of them has an extra mutation that lead to development of another lymphoma cell thus forming composite lymphoma [6]. Dachman et al. [7] was the first to define primary splenic lymphoma as a lymphoma involving only the spleen and the splenic hilar lymph nodes, but not the liver or other sites. A period of six months or more between detection of splenic involvement and appearance of lymphoma elsewhere is also required. Another definition of primary splenic lymphoma was proposed by Dachman et al. [7] that suggested the diagnosis of Primary splenic lymphoma for any lymphoma with splenic involvement and splenomegaly as a predominant feature that was not present in our patient. Quayle et al. [8] then defined Primary Splenic lymphoma as, lymphoma presenting with splenomegaly, at least bicytopenia, and in the absence of peripheral adenopathy. Our patient did not fit the latter definition as the presentation did not include splenomegaly and no evidence of cytopenia. Thus appropriate strict definition of primary splenic lymphoma is still controversial [7]. Our patient fulfilled the inclusion criteria of cohort of primary splenic lymphoma published by Dachman et al. [7], with the spleen being the only organ involved at time of demonstration with only direct extension of the disease is allowed and negative lymph nodes and marrow examination. Similar to most patients, pathology revealed scattered whitish solid masses of varied sizes. But our patient was like just 1 patient out of the 23 patients, had a normal sized spleen [8]. Lymphomas of the GI are extremely rare conditions with the cecum as the most common site of involvement. IT accounts less than $1 \%$ of colorectal cancers. Lymphomas of the gastrointestinal tract present similar to carcinomas of the involved site (anemia, abdominal pain, weight loss) [9] also association of lymphoma with adenoma has been described and rarely present. GI lymphomas, especially primary Hodgkin lymphoma of the gastrointestinal tract, are usually associated with immunodeficiency or inflammatory bowel disease. Our patient had verruca valgaris that can explain a state of immunodeficiency and cytopathology showed underlying EBV infection [10]. Mucosa associated lymphomas with marginal zone lymphoma and $\mathrm{T}$ cell lymphomas had been detected in stomach in previous reports [11]. Our case is lymphoma stage IIIB with lymph nodes involvement but the initial presentation was the extra nodal involvement mimicking colorectal carcinomas. To our knowledge it's the 2nd composite lymphoma of Hodgkin and NonHodgkin involving the colon. Very few cases of composite lymphoma have been reported involving the GI tract; about 2 cases in the upper GI and 2 cases in the lower GI tract [11]. Composite lymphomas of the classical Hodgkin lymphoma and large B-cell lymphoma are rare and the reported cases are around 20 cases only at different sites of involvement [11]. Treatment of composite lymphoma has never been standardized due to the rarity of the case. Therapeutic approach depends on type of composite lymphomas. It is logical to treat as Hodgkin lymphoma if it is present with indolent $\mathrm{B}$ cell lymphoma where anti CD 20 can be added concurrently or sequentially. If Hodgkin lymphoma is present with diffuse large $\mathrm{B}$ cell lymphoma aggressive treatment is recommended. Radiotherapy is added according to the stage and is case specific decision.

\section{References}

1. Gobbi PG, Grignani GE, Pozzetti U, Bertoloni D, Pieresca C, Montagna G (1994) Primary splenic lymphoma; does it exist? Haematologica 79: 286-293.

2. Cavanna L, Artioli F, Vallisa D, Di Donato C, Bertè R, et al. (1995) Primary lymphoma of the spleen. Report of a case with diagnosis by fine needle guided biopsy. Heamatologica 80: 241-243.

3. Kim JK, Hahn JS, Kim GE, Yang W (2005) Three Cases of Diffuse Large B-Cell Lymphoma Presenting as Primary Splenic Lymphoma. Yonsei Medical Journal 46: 703-709.

4. Singh M, Mohanvir K, Nishit G, Bodal VK (2014) Primary Splenic Hodgkin's Lymphoma: A Case Report, Research and Reviews: Journal of Medical and Health Sciences 3: 37-41.

5. Grangeiro MP, Magalhães SM, Ferreira FV, Filho NF (2004) Simultaneous occurrence of follicular lymphoma and mixed-cellularity Hodgkin's lymphoma: lymph node and extranodal involvement. Journal Brasil Pathology Medical Lab 40: $33-36$

6. Küppers R, Dührsen U, Hansmann M (2014) Pathogenesis, diagnosis, and treatment of composite lymphomas. Lancet Oncology, pp: 435-446.

7. Dachman AH, Buck JL, Krishnan J, Aguilera NS, Buetow PC (1998) Primary Non-Hodgkin's Splenic Lymphoma. Clinical Radiology 53: 137-142.

8. Quayle FJ, Lowney JK (2006) Colorectal Lymphoma. Clinics In Colon and Rectal Surgery 19: 49-53.

9. Valbuena JR, Gualco G, Espejo-Plascencia I, Medeiros LJ (2005) Classical Hodgkin lymphoma arising in rectum. Heart, Lung and Circulation 9: 38-42.

10. Kyoung YW, Young-Hyeh K, Dae-Shick K, Won-Seog K, Poong-Lyul R, et al (2008) Composite marginal zone B cell lymphoma and enteropathy-type T cell lymphoma of the stomach: a case report. European Journal of Gastroenterology and Hepatology 20: 791-795.

11. Goyal G (2016) Composite lymphoma with diffuse large B-cell lymphoma and classical Hodgkin lymphoma components: A case report and review of the literature, Pathology Research and Practice 212: 1179-1190. 